Supporting information

\title{
A super-stability luminescent lanthanide metal organic gel utilized in electrochemiluminescence sensor for epinephrine detection with a narrow potential sweep range
}

\footnotetext{
Cun Wang ${ }^{\dagger, \star, ~ Q i a n ~ H a n ~}{ }^{\dagger, \S}$, Pingkun Liu ${ }^{\dagger}$, Gui Zhang ${ }^{\dagger}$, Li Song ${ }^{\dagger}$, Xiaochuan Zou ${ }^{\dagger}$, Yingzi Fu*†

${ }^{\dagger}$ Key Laboratory of Luminescence Analysis and Molecular Sensing (Southwest University), Ministry of Education, School of Chemistry and Chemical Engineering, Southwest University, Chongqing 400715, China.

$\$$ Chongqing Collaborative Innovation Center for Functional Food, Chongqing University of Education, Chongqing 400067, China.

$\S$ Laboratory of Environment Change and Ecological Construction of Hebei Province, College of Resources and Environment Science, Hebei Normal University, Shijiazhuang, Hebei 050024, China.
}

Corresponding author: Yingzi Fu, Tel: +86-023-68252277, E-mail address: fyzc@,swu.edu.cn ORCID: Yingzi Fu: 0000-0003-4894-8549 
Table of contents

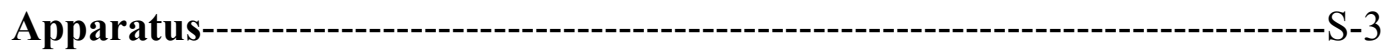

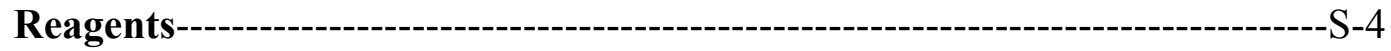

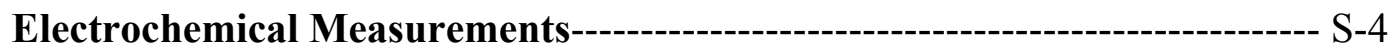

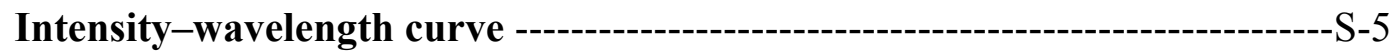

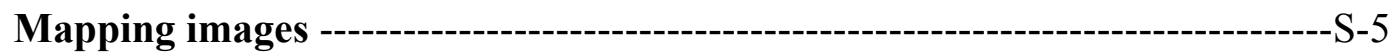

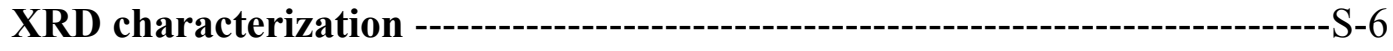

Electrochemical characterization of different modified electrodes-------------S-6

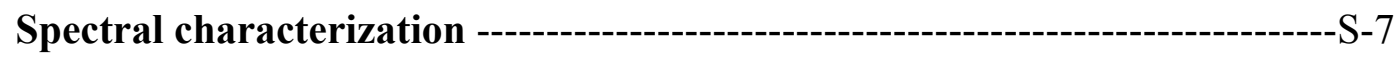

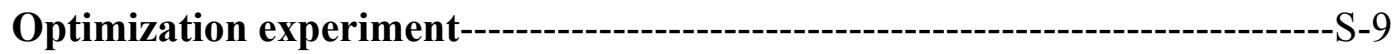

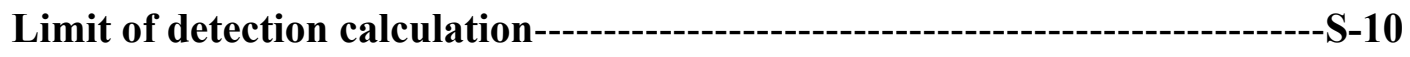

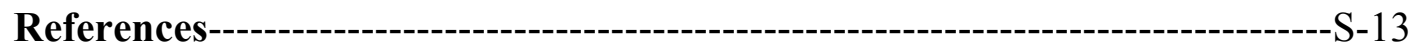




\section{Apparatus}

Electrochemiluminescence (ECL) and electrochemistry measurements were carried out on a model MPI-A electrochemiluminescence analyzer (Xi' An Remax Electronic Science \& Technology Co. Ltd., Xi' An, China) and a CHI 660E electrochemical workstation (Shanghai Chenhua Instruments, China), respectively. The three-electrode system was used for ECL and electrochemistry measurements with a glassy carbon electrode (GCE, $\Phi=4.0 \mathrm{~mm}$ ) as the working electrode, a platinum wire as the counter electrode, and an $\mathrm{Ag} / \mathrm{AgCl}$ (saturated $\mathrm{KCl}$ ) as the reference electrode. X-ray photoelectron spectroscopy (XPS) was performed using a thermos escalab 250Xi spectrometer $(0 \sim 5000 \mathrm{eV}$, Thermo Electron Corporation, America). Transmission 
electron microscope (TEM, Talos F200S, America) with an accelerating voltage of 200 $\mathrm{KV}$, scanning electron microscope (SEM, HitachiInstruments Co., Japan) and an Oxford-INCA energy dispersive X-ray (EDX) spectroscope were used to determine the appearance and the elemental composition of materials. The ultraviolet-visible (UVVis) spectrum was obtained from a UV-2600 spectrophotometer (Shimadzu, Japan). The Fourier transform infrared spectrum (FTIR) was recorded with a Fourier Transform Infrared Spectrometer (PerkinElmer, USA) in the range of 4000-400 $\mathrm{cm}^{-1}$ using the $\mathrm{KBr}$ assisted tableting method at the room temperature. The fluorescence (FL) spectrum was performed on a RF-5301PC spectrophotometer (Shimadzu, Tokyo, Japan). Dynamic light scattering (DLS) was carried out on Zetasize Nano ZS (Malvern, United Kingdom). The ECL emission spectra were obtained on an electrochemical workstation with a Newton EMCCD spectroscopy detector (Andor Co., Tokyo, Japan). The time interval was set to $0.1 \mathrm{~s}$, the potential scan ranged from 0.00 to $-0.85 \mathrm{~V}$. All measurements were carried out at the room temperature $\left(25 \pm 1^{\circ} \mathrm{C}\right)$.

\section{Reagents}

Tris(4,4'-dicarboxylicacid-2,2'-bipyridyl) ruthenium (II) dichloride (Ru(dcbpy) ${ }_{3}{ }^{2+}$, 98\%), Tris(2,2'-bipyridyl) ruthenium (II) dichloride $\left(\mathrm{Ru}(\mathrm{bpy}) 3_{3}{ }^{2+}, 98 \%\right)$ and $4^{\prime}-(4-$ carboxyphenyl)-2,2':6',2"-terpyridine (Hcptpy, 97\%) were acquired from Suna Tech Inc. (Jiangsu, China). Terbium(III) nitrate hexahydrate $\left(\mathrm{Tb}\left(\mathrm{NO}_{3}\right)_{3} \cdot 6 \mathrm{H}_{2} \mathrm{O}, 99.99 \%\right)$, Triethylamine ( $\geq 99.5 \%$ ), uric acid (99\%), ascorbic acid (99.9\%), glucose (99.5\%), Lcysteine (98\%), L-proline (99\%), L-tryptophan (98\%), L-alanine ( $\geq 99.5 \%$ ), L-arginine 
(99\%) were gained from J\&k Chemical Co. (Beijing, China). Tris-HCl buffer (20 $\mathrm{mmol} \cdot \mathrm{L}^{-1}, \mathrm{pH} 7.0$ ) was used as the experimental test solution. All other chemicals were analytical grade. Double distilled water was used.

\section{Electrochemical Measurements}

The cyclic voltammetry (CV) and electrochemical impedance spectroscopy (EIS) measurements were conducted in $5 \mathrm{mmol} \cdot \mathrm{L}^{-1}\left[\mathrm{Fe}(\mathrm{CN})_{6}\right]^{3-/ 4-}\left(20 \mathrm{mmol} \cdot \mathrm{L}^{-1} \mathrm{Tris}-\mathrm{HCl}, \mathrm{pH}\right.$ 7.0). The potential sweep ranged from -0.20 to $0.60 \mathrm{~V}$ with a scan rate of $50 \mathrm{mV} \mathrm{s}^{-1}$. The ECL signals were recorded in $2.0 \mathrm{mmol} \cdot \mathrm{L}^{-1} \mathrm{~K}_{2} \mathrm{~S}_{2} \mathrm{O}_{8}\left(20 \mathrm{mmol} \cdot \mathrm{L}^{-1} \mathrm{Tris}-\mathrm{HCl}\right.$, $\mathrm{pH}$ 7.0). The potential sweep ranged from 0.00 to $-0.85 \mathrm{~V}$ with a scan rate of $100 \mathrm{mV} \cdot \mathrm{s}^{-1}$ and the time interval was $0.1 \mathrm{~s}$. The photomultiplier tube (PMT) was set at $600 \mathrm{~V}$.

\section{Intensity-wavelength curve}

The intensity-wavelength curve is based on the following formula. ${ }^{1}$

$$
I=\frac{\mathrm{Nh}}{\lambda \mathrm{At}}
$$

Here, I, $\lambda, \mathrm{h}, \mathrm{A}, \mathrm{t}$ and $\mathrm{N}$ represent the intensity, wavelength, Planck constant, irradiation area, time and the total number of photons reaching on A in time interval t.

\section{Mapping images}




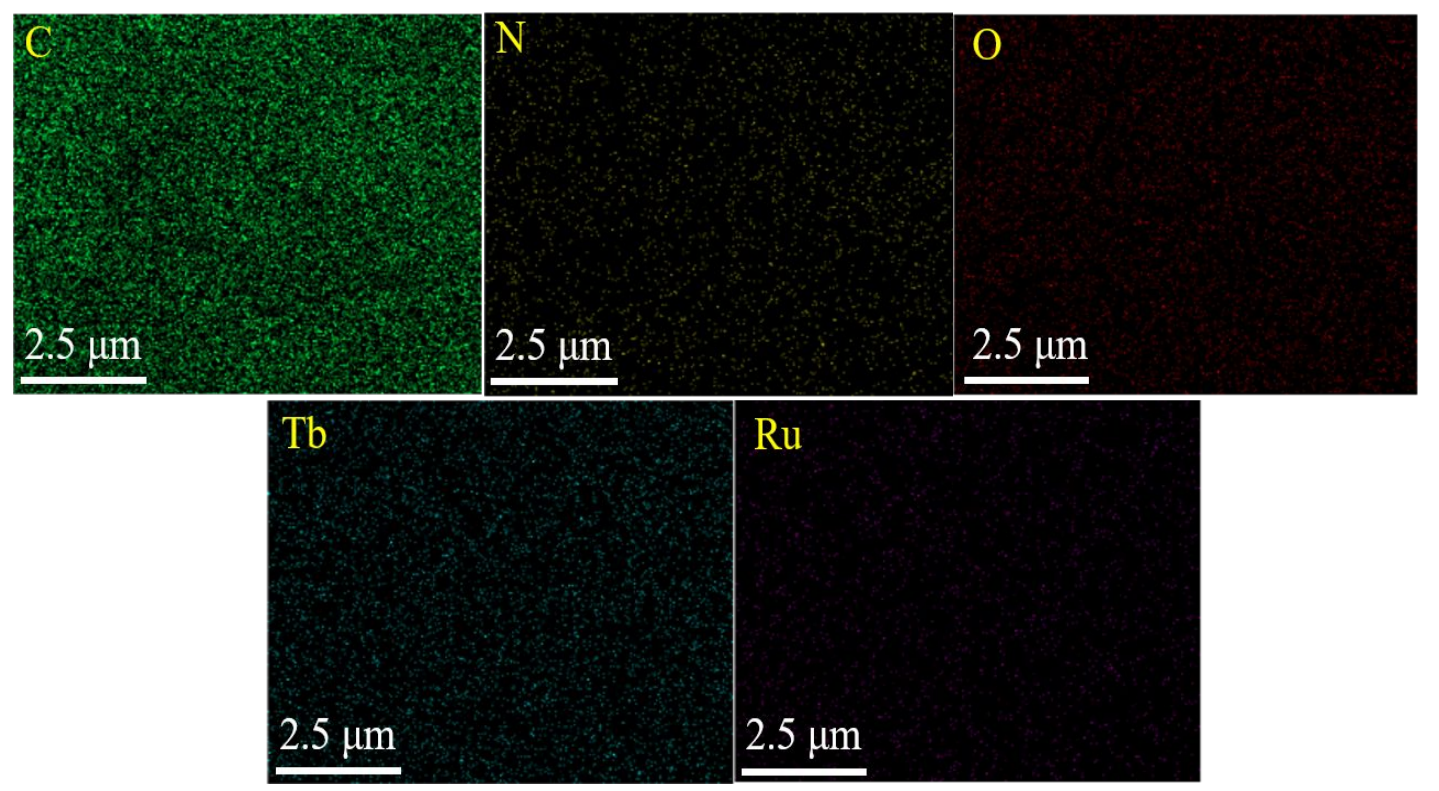

Figure S1. Mapping images of Tb-Ru-MOG

\section{XRD characterization}

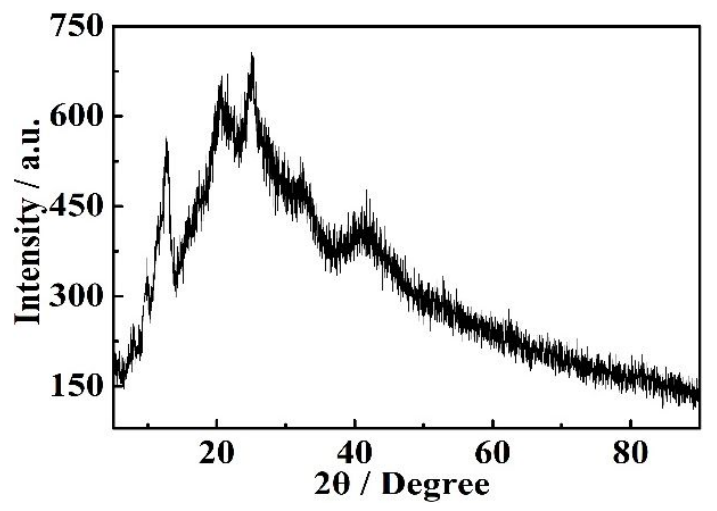

Figure S2. The XRD pattern of the Tb-Ru-MOG

Electrochemical characterization of different modified electrodes 
Cyclic voltammetry (CV) and electrochemical impedance spectroscopy (EIS) were used to characterize the electrode modification process. As shown in the Fig. S3A, compared with bare GCE (curve a) showing two reversible redox peaks, the redox current of Tb-Ru-MOG modified GCE (curve b) decreased. At the same time, when 1.0 $\times 10^{-4} \mathrm{~mol} \cdot \mathrm{L}^{-1} \mathrm{EP}$ was added in $5.0 \mathrm{mmol} \cdot \mathrm{L}^{-1}\left[\mathrm{Fe}(\mathrm{CN})_{6}\right]^{3-/ 4-}$ solution, the redox current of Tb-Ru-MOG/GCE also decreases. It suggests that Tb-Ru-MOG and EP hinder electron transfer. From the Fig.S3B, the electron transfer resistance (Ret) value of the bare GCE is relatively small, which is due to the free electron-transfer process. However, after Tb-Ru-MOG was modified on GCE, the Ret value increases significantly. The Ret value of Ru-MOG/GCE also increases after $1.0 \times 10^{-4} \mathrm{~mol} \cdot \mathrm{L}^{-1}$ EP was added in $5.0 \mathrm{mmol} \cdot \mathrm{L}^{-1}\left[\mathrm{Fe}(\mathrm{CN})_{6}\right]^{3-/ 4-}$ solution. The $\mathrm{CV}$ results are corroborated by the EIS, indicating the electrode has been successfully prepared.
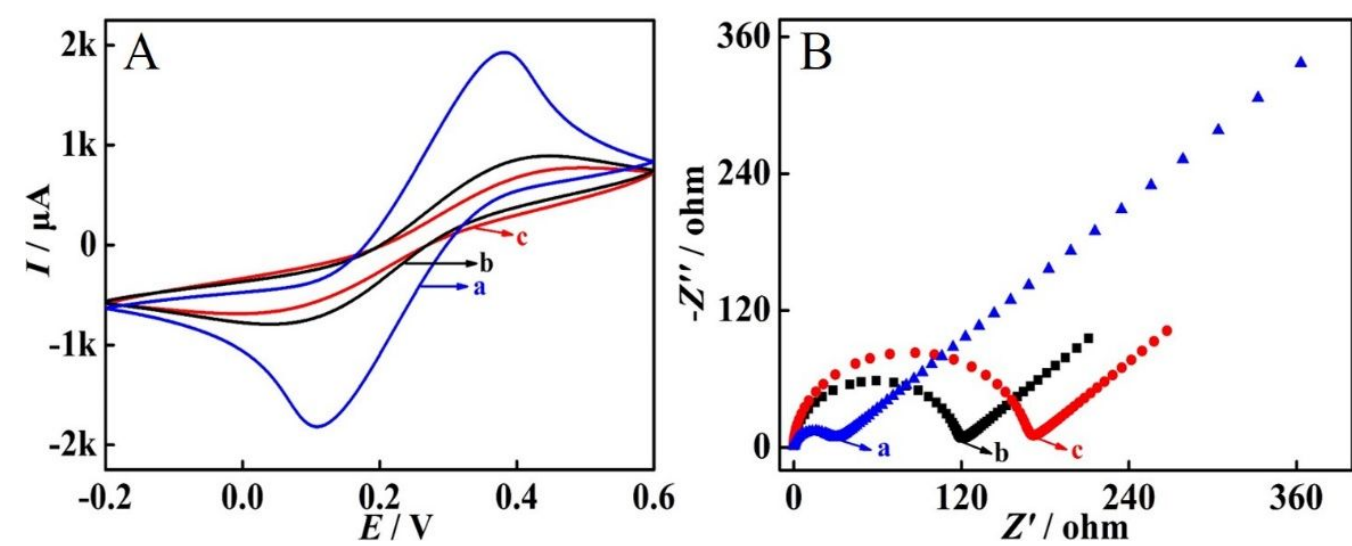

Figure S3. (A) CV and (B) EIS responses of different modified electrodes in 5.0 $\mathrm{mmol} \cdot \mathrm{L}^{-1}\left[\mathrm{Fe}(\mathrm{CN})_{6}\right]^{3-/ 4-}$ : (a) bare GCE, (b) Tb-Ru-MOG/GCE, and (c) Tb-RuMOG/GCE (containing $\left.1.0 \times 10^{-4} \mathrm{~mol} \cdot \mathrm{L}^{-1} \mathrm{EP}\right)$. 


\section{Spectral characterization}

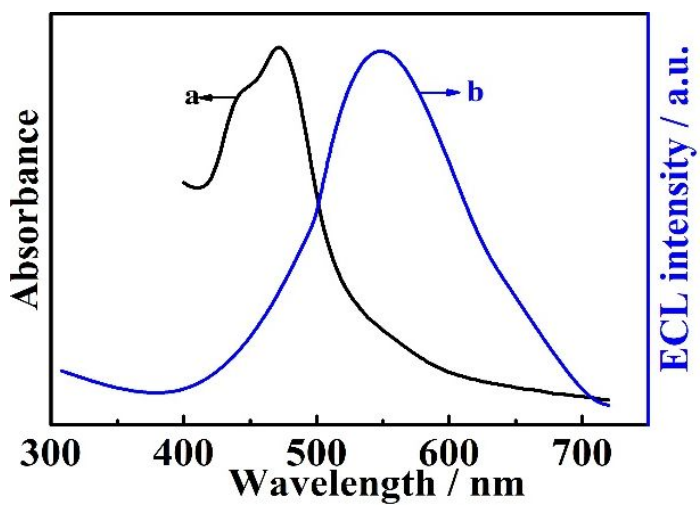

Figure S4. (a) The UV-vis absorption spectrum of Tb-Ru-MOG and (b) the ECL spectrum of $\mathrm{Tb}-\mathrm{MOG} / \mathrm{GCE}$.
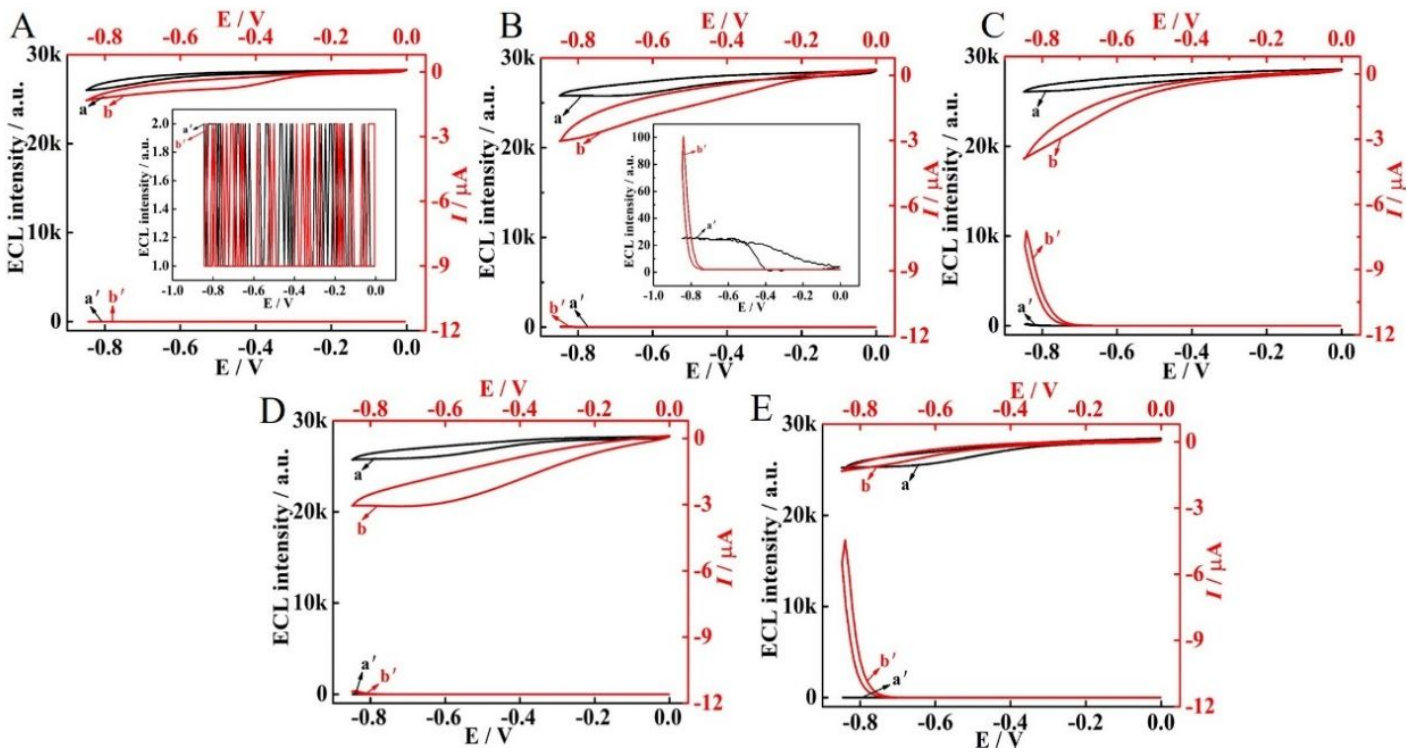

Figure S5. The CV and ECL behavior of (A) Bare GCE, (B) Ru(dcbpy $)_{3}{ }^{2+},(\mathrm{C}) \mathrm{Tb}-$

Ru-NCP, (D) Tb-MOG and (E) Tb-Ru-MOG in (a, a') absence and (b, b') presence of

$2.0 \mathrm{mmol} \cdot \mathrm{L}^{-1} \mathrm{~S}_{2} \mathrm{O}_{8}{ }^{2-}\left(20 \mathrm{mmol} \cdot \mathrm{L}^{-1}\right.$ Tris- $\left.\mathrm{HCl}, \mathrm{pH} 7.0\right)$. 

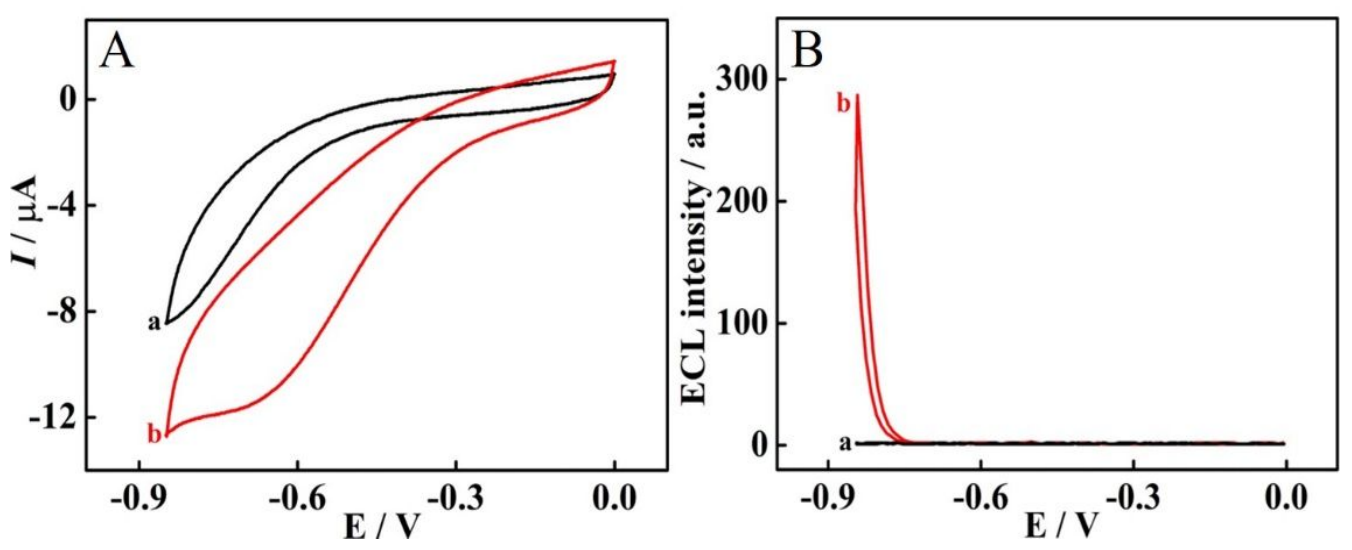

Figure S6. (A) Cyclic voltammograms (CVs) and (B) ECL curves in $20 \mathrm{mmol} \cdot \mathrm{L}^{-1}$ Tris-HCl (pH 7.0): (a) bare GCE and (b) Tb-Ru-MOG/GCE
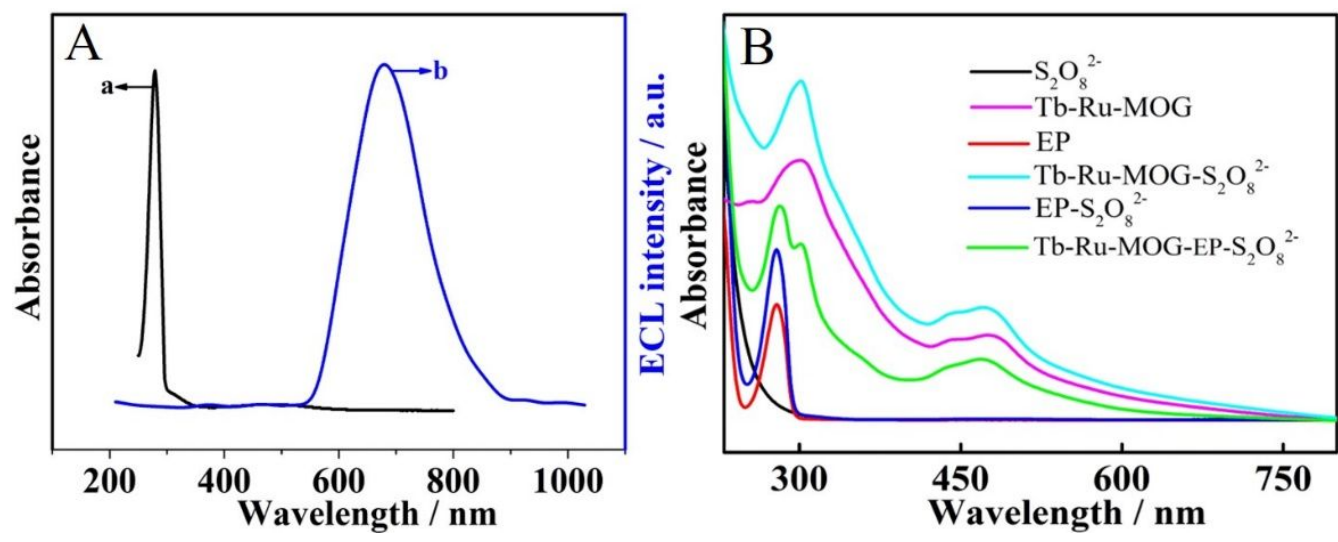

Figure S7. (A) the UV-vis absorption of EP (a) and the 2D ECL spectrum of Tb-RuMOG (b), (B) FTIR spectra of $\mathrm{S}_{2} \mathrm{O}_{8}{ }^{2-}$, Tb-Ru-MOG, EP, Tb-Ru-MOG and $\mathrm{S}_{2} \mathrm{O}_{8}{ }^{2-}$ (TbRu-MOG- $\left.\mathrm{S}_{2} \mathrm{O}_{8}{ }^{2-}\right)$, EP and $\mathrm{S}_{2} \mathrm{O}_{8}{ }^{2-}\left(\right.$ EP- $\left.\mathrm{S}_{2} \mathrm{O}_{8}{ }^{2-}\right)$, mixture of Tb-Ru-MOG, EP and $\mathrm{S}_{2} \mathrm{O}_{8}{ }^{2-}$ (Tb-Ru-MOG-EP-S $\mathrm{O}_{8}{ }^{2-}$ ). 


\section{Optimization experiment}

As can be seen from the Figure S8, with increasing of the $\mathrm{pH}$ values, the ECL signals decrease. The decrease in the ECL intensity at high $\mathrm{pH}$ values was attributed to the oxygen evolution reaction at the electrode, which is shifted to less positive potentials with increasing $\mathrm{pH}^{2}$ On the other hand, with the increases of $\mathrm{pH}$ value, the concentration of $\mathrm{OH}^{-}$increases, which can react with the sulfate radical anion $\left(\mathrm{SO}_{4}{ }^{-}\right.$, generated during reduction of $\mathrm{S}_{2} \mathrm{O}_{8}{ }^{2-}$ ) according to the following Eq: ${ }^{3}$

$\mathrm{SO}_{4}{ }^{-\bullet}+\mathrm{OH}^{-} \rightarrow \mathrm{SO}_{4}^{2-}+\cdot \mathrm{OH}$

So the decrease of $\mathrm{SO}_{4}{ }^{-\bullet}$ concentration leads to the decrease of ECL intensity.

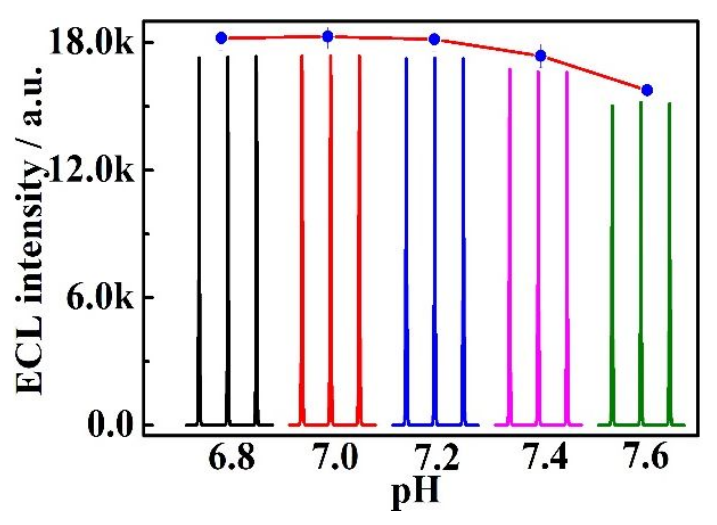

Figure S8. The effect of the $\mathrm{pH}$.

The dosage of Tb-Ru-MOG nanocomposite is optimized. As displayed in Figure S9, the ECL signal increases along with the extension of the added amount of Tb-Ru-MOG 
$\left(1.5 \mathrm{mg} \cdot \mathrm{mL}^{-1}\right)$, and overflows till $6 \mu \mathrm{L}$. Therefore, $5 \mu \mathrm{L}$ is chosen for the further experiments.

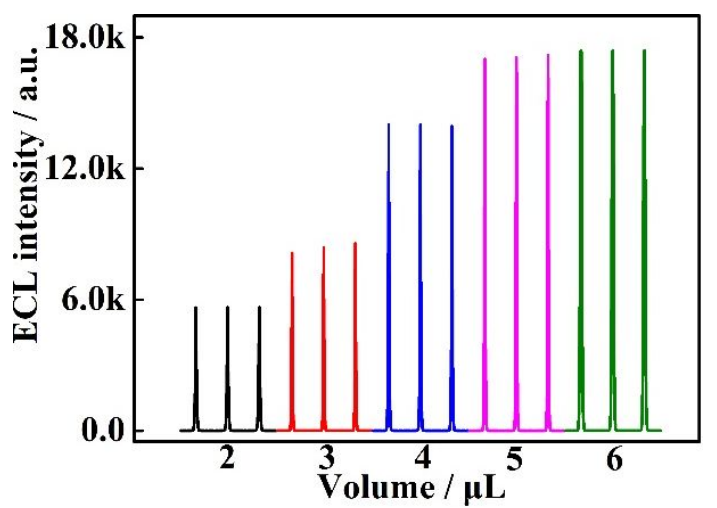

Figure S9. Optimization of the proposed sensor with different dosage of Tb-Ru-MOG $\left(1.5 \mathrm{mg} \cdot \mathrm{mL}^{-1}\right)$.

\section{Limit of detection calculation}

The limit of detection (LOD) has been calculated according to the following equation: ${ }^{10,11}$

$$
I_{\mathrm{L}}=I_{\mathrm{B}}+\mathrm{k}_{1} \mathrm{~S}_{\mathrm{B}}
$$

An ECL measurement for blank samples was executed with three parallel tests, which exhibited average ECL intensity of 17364.73 a.u. with standard deviation $\left(\mathrm{S}_{\mathrm{B}}\right)$ of 17.38. On the one hand, the signal-to-noise ratio value $\left(\mathrm{k}_{1}\right)$ of 3 , which allows a confidence level of $99.86 \%$. On the other hand, the changes in the ECL intensities $\left(\Delta I_{\mathrm{ECL}}\right)$ were found to be linearly depended on the logarithm of EP concentration, so the $I_{\mathrm{B}}=0$. The smallest detectable signal can be calculated as

$$
I_{\mathrm{L}}=I_{\mathrm{B}}+\mathrm{k}_{1} \mathrm{~S}_{\mathrm{B}}=0+3 \times 17.38=52.14
$$

According to the linear regression equation $\Delta I=24812.7+2408.5 \lg c$, the LOD could be calculated as $5.2 \times 10^{-11} \mathrm{~mol} \cdot \mathrm{L}^{-1}$. 


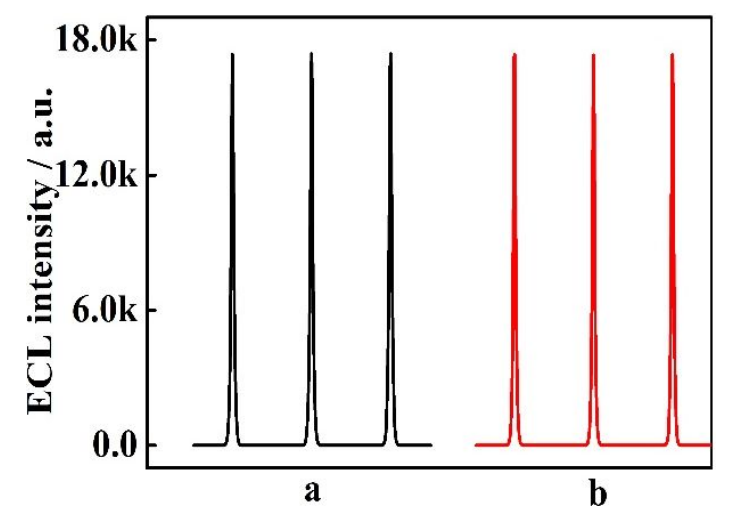

Figure S10. ECL responses of the Tb-Ru-MOG/GCE (a) on the day of preparation and (b) after three months of storage.

Table S1.

Table S1. Different methods for the detection of EP

\begin{tabular}{|c|c|c|c|}
\hline Analytical methods & $\begin{array}{l}\text { detection range } \\
\left(\mathrm{mol} \cdot \mathrm{L}^{-1}\right)\end{array}$ & $\begin{array}{l}\text { detection limit } \\
\left(\mathrm{mol} \cdot \mathrm{L}^{-1}\right)\end{array}$ & references \\
\hline Fluorescence & $1.0 \times 10^{-5}-1.0 \times 10^{-4}$ & $5.9 \times 10^{-10}$ & 4 \\
\hline Capillary electrophoresis & $4.0 \times 10^{-8}-5.0 \times 10^{-6}$ & $9.0 \times 10^{-9}$ & 5 \\
\hline Electrochemistry & $6.5 \times 10^{-9}-4.1 \times 10^{-5}$ & $1.1 \times 10^{-10}$ & 6 \\
\hline Electrochemistry & $1.0 \times 10^{-7}-7.5 \times 10^{-5}$ & $1.3 \times 10^{-8}$ & 7 \\
\hline Chemiluminescence & $1.0 \times 10^{-7}-7.1 \times 10^{-3}$ & $1.1 \times 10^{-9}$ & 8 \\
\hline Electrochemiluminescence & $8.0 \times 10^{-10}-2.0 \times 10^{-7}$ & $1.0 \times 10^{-10}$ & 9 \\
\hline Electrochemiluminescence & $1.0 \times 10^{-10}-1.0 \times 10^{-3}$ & $5.2 \times 10^{-11}$ & This work \\
\hline
\end{tabular}


Table S2.

Table S2. Recovery results of the proposed sensor in EP hydrochloride injection and healthy human serum

\begin{tabular}{|c|c|c|c|c|c|}
\hline Sample number & $\begin{array}{l}\text { Concentration } \\
\left(\mathrm{mol} \cdot \mathrm{L}^{-1}\right)\end{array}$ & $\begin{array}{l}\text { Added } \\
\left(\mathrm{mol} \cdot \mathrm{L}^{-1}\right)\end{array}$ & $\begin{array}{l}\text { Found } \\
\left(\mathrm{mol} \cdot \mathrm{L}^{-1}\right)\end{array}$ & $\begin{array}{l}\text { Recovery } \\
(\%)\end{array}$ & $\begin{array}{l}\text { RSD } \\
(\%)\end{array}$ \\
\hline EP hydrochloride injection $1^{\mathrm{a}}$ & $1.0 \times 10^{-3}$ & - & $1.01 \times 10^{-3}$ & 101.0 & 0.4 \\
\hline EP hydrochloride injection $2^{\mathrm{b}}$ & $1.0 \times 10^{-8}$ & - & $1.02 \times 10^{-8}$ & 102.0 & 3.9 \\
\hline Healthy human serum $1^{\mathrm{c}}$ & - & $1.0 \times 10^{-4}$ & $1.01 \times 10^{-4}$ & 101.0 & 4.9 \\
\hline Healthy human serum $2^{c}$ & - & $1.0 \times 10^{-5}$ & $1.00 \times 10^{-5}$ & 100.0 & 3.3 \\
\hline Healthy human serum $3^{c}$ & - & $1.0 \times 10^{-6}$ & $9.81 \times 10^{-7}$ & 98.1 & 3.0 \\
\hline
\end{tabular}

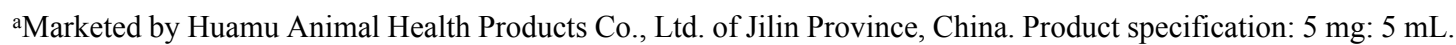
bMarketed by Quanyu Sheng Wu Ke Ji Animal Pharmaceutical Co., Ltd of Zhuma Dian Province, China. Product specification: $1 \mathrm{mg}: 1 \mathrm{~mL}$.

'Provided by Ninth People's Hospital of Chongqing, China.

\section{References}

(1) Xiong, C. Y.; Wang, H. J.; Liang, W. B.; Yuan, Y. L.; Yuan, R.; Chai, Y. Q. Luminescence-functionalized metal-organic frameworks based on a ruthenium(II) complex: A signal amplification strategy for electrogenerated chemiluminescence Immunosensors. Chem. Eur. J., 2015, 21, 9825-9832. 
(2) Rubinstein, I., Bard, A. J. Electrogenerated chemiluminescence. 37. Aqueous ecl systems based on $\mathrm{Ru}\left(2,2^{\prime} \text {-bipyridine }\right)_{3}{ }^{2+}$ and oxalate or organic acids, J. Am. Chem. Soc. 1981, 103, 512-516.

(3) Huang W. Y.; Liu F.; Lu A. H.; Wang F. T.; Miao D. R., Removal of Organic Pollutants by Hydrogen Peroxide and Sodium Persulfate: A Review, Environ. Sci. Technol., 2013, 36, 88-95.

(4) Govindaraju, S.; Reddy, A. S.; Kim, J.S.; Yun, K. Sensitive detection of epinephrine in human serum via fluorescence enhancement of gold nanoclusters. Appl. Surf. Sci., 2019, 498, 143837-143846.

(5) Zhao, Y.S.; Zhao, S.L.; Huang, J.M.; Ye, F.G. Quantum dot-enhanced chemiluminescence detection for simultaneous determination of dopamine and epinephrine by capillary electrophoresis. Talanta, 2011, 85, 2650-2654,

(6) Fatma, S.; Prasad, B. B.; Jaiswal, S; Singh, R; Singh, K. Electrochemical simultaneous analysis of dopamine and epinephrine using double imprinted one monomer acryloylated graphene oxide-carbon black composite polymer. Biosens. Bioelectron., 2019, 135, 36-44.

(7) Avcı, O.; Perk, B.; Varol, T. O.; Buyu“ksünetçi, Y. T.; Hakli, O.; Anik, U. A polyoxy group branched diazo dye as an alternative material for the fabrication of an electrochemical epinephrine sensor. New J. Chem., 2019, 43, 18575-18581.

(8) Qiu, H.M.; Luo, C.N; Sun, M.; Lu, F.G.; Fan, L.L.; Li, X.J. A chemiluminescence sensor for determination of epinephrine using graphene oxide-magnetite-molecularly imprinted polymers. Carbon, 2012, 50, 4052-4060. 
(9) Zheng, R. J.; Zhao, C. H.; Zhong, J. H.; Qiu, Z. H.; Hu, Z. B. Int. Determination of epinephrine using a novel sensitive electrochemiluminescence sensor based on $\mathrm{ZnO}$ nanoparticles modified pencil graphite electrode. J. Electrochem. Sci., 2019, 14, 9380-9390.

(10)Long, G.; Winefordner, J. Limit of detection. A closer look at the IUPAC definition, Anal. Chem., 1983, 55, 712A-724A.

(11)Jiang, M. H.; Lu, P.; Lei, Y. M.; Chai, Y. Q.; Yuan, R.; Zhuo, Y., Self-accelerated Electrochemiluminescence Emitters of $\mathrm{Ag} @ \mathrm{SnO}_{2}$ Nanoflowers for Sensitive Detection of Cardiac troponin T, Electrochim. Acta, 2018, 271, 464-471. 